\title{
Predictors of Social Functioning in Patients with Higher and Lower Levels of Reduced Emotional Experience: Social Cognition, Social Competence, and Symptom Severity
}

\author{
Philip D. Harvey, PhD ${ }^{1,2}$, Elizabeth Deckler, BA ${ }^{1}$, L. Fredrik Jarskog, MD ${ }^{3}$, David L. Penn, \\ $\mathrm{PhD}^{4,5}$, and Amy Pinkham, $\mathrm{PhD}^{6,7}$ \\ ${ }^{1}$ Department of Psychiatry and Behavioral Sciences, University of Miami Miller School of \\ Medicine, Miami, FL \\ ${ }^{2}$ Research Service, Miami VA Healthcare System \\ ${ }^{3}$ Department of Psychiatry, University of North Carolina, Chapel Hill, NC \\ ${ }^{4}$ Department of Psychology, University of North Carolina, Chapel Hill, NC \\ ${ }^{5}$ School of Psychology, Australian Catholic University, Melbourne, VIC \\ ${ }^{6}$ School of Behavioral and Brain Sciences, The University of Texas at Dallas, Richardson, TX. \\ ${ }^{7}$ Department of Psychiatry, University of Texas Southwestern Medical School, Dallas, TX.
}

\section{Abstract}

Background.-Deficits in social functioning in schizophrenia are primarily predicted by negative symptoms, social cognition deficits, and social skills deficits. Here we examine those predictive variables across variations in the severity of reduced emotional experience. We hypothesized that in patients with high symptom severity, factors such as social cognition would have reduced importance for predicting social outcomes.

Methods.-Participants with schizophrenia $(n=312)$ were tested using five different measures of social cognition. Performance-based assessments and clinical ratings of reduced emotion experience were used to assess social competence. High contact informants rated interpersonal

\footnotetext{
Correspondence: Philip D. Harvey, PhD, Miller School of Medicine, University of Miami, 1120 NW $14^{\text {th }}$ Street, Suite 1450 , Miami, FL 33136 US (telephone: 305-243-4094; fax: 305-243-1619; philipdharvey1@ cs.com. Contributions of the Authors.

Drs. Harvey, Penn, and Pinkham designed the study and supervised its conduct

Ms. Deckler analyzed the data and wrote several drafts of the paper. All Authors reviewed these drafts.

All authors have approved the final version.

Publisher's Disclaimer: This is a PDF file of an unedited manuscript that has been accepted for publication. As a service to our customers we are providing this early version of the manuscript. The manuscript will undergo copyediting, typesetting, and review of the resulting proof before it is published in its final citable form. Please note that during the production process errors may be discovered which could affect the content, and all legal disclaimers that apply to the journal pertain.

Conflict Of Interest Statement.

Dr. Harvey has received consulting fees or travel reimbursements from Akili, Boehringer Ingelheim, Intra Cellular Therapeutics, OtsukaAmeica, Sanofi Pharma, Sunovion Pharma, Takeda Pharma, and Teva Pharma during the past year. He has a research grant from Takeda and from the Stanley Medical Research Foundation.

The other authors have no biomedical conflicts of interest.
} 
functioning and social acceptability of behavior, while unaware of other patient data. Patients were divided into higher and lower reduced emotional experience using previously validated criteria.

Results. $-33 \%$ of the patients had at least moderate symptoms of reduced emotional experience. Patients with greater severity had more social functioning impairment, but not poorer social competence and social cognition. In the patients with lower severity, social cognition accounted for $9 \%$ of the variance in interpersonal functioning, while in patients with higher severity, social cognition did not predict any variance. In the patients with lower severity, social cognition accounted for $4 \%$ of the variance in social acceptability of behavior, while in patients with higher severity, social cognition also did not predict any variance.

Implications.-The influence of social cognition on social outcomes appears greater in patients with less severe symptoms of reduced emotional experience. As there are treatments for both these symptoms and social cognition with demonstrated efficacy, these data suggest differential application of these interventions based on symptom severity.

\section{Introduction}

People with schizophrenia have impaired everyday functioning, with these impairments spanning domains of vocational, social, and everyday activities (Galderisi et al., 2014). While neurocognitive deficits and associated impairments in functional capacity have been identified as primary predictors of limitations in work and everyday activities, negative symptoms (Strassnig et al., 2015), particularly when combined with social cognitive deficits (Kalin et al., 2015) have been found to be primary predictors of patients' real-world social outcomes. Further, negative symptoms are multidimensional, with reductions in emotion expression and reductions in emotional experience (often referred to as avolition-apathy) having different functional implications (Strauss et al., 2013). For example, reduced emotional experience has been found to correlate, at both lower and higher levels of severity, with impairments in social functioning while reduced emotional expression manifested minimal correlations with social functioning (Harvey et al., 2017; Strassnig, et al., 2018).

There are other determinants of social outcomes in addition to social cognition and reduced emotional experience. Primary among them is social competence, or social skills (Kalin et al., 2015). There has been a long history of study of social competence in people with schizophrenia, focusing on both defining the limitations and treating deficits. In our previous work, we reported that negative symptoms accounted for a considerably greater percentage of the variance in everyday social outcomes than social skills that appeared to contribute minimally (Robinson et al., 2013). In a later study, (Kalin et al., 2015) we found that the combination of social cognitive performance and negative symptoms accounted for $32 \%$ of the variance in a performance-based measure of social competence. Further, when social competence, social cognition, and negative symptoms were used to predict social outcomes, it was also found that the same amount of variance, $32 \%$, was shared with social outcomes. Only two of the PANSS negative symptoms predicted everyday social outcomes, passiveapathetic social withdrawal and active social avoidance, and these symptoms were found to load on a factor reflecting reduced emotional experience in a very large sample study ( $\mathrm{n}=6889$; Khan et al., 2017). 
These results suggest a combined role of social cognition, social competence, and social motivation as the primary predictors of everyday social outcomes. However, reduced emotional experience symptoms vary widely in their severity. For example, individuals with schizophrenia related conditions such as schizotypal personality disorder and attenuated psychosis syndrome have milder version of these symptoms (Raine, 2006; Carrion et al., 2016). Importantly, more substantial deficits in social motivation could easily lead to poor social outcomes in the presence of intact social skills or social cognition, simply because of reduced attempts to engage in any social activities. Hence, the role of social competence and social cognition in the predictions of social outcomes could be hypothesized to be dependent on the level of severity of reduced emotional experience. Minimal symptom severity might be expected to render levels of social cognitive abilities and social competence more important for prediction. Moderate or more severe symptoms could lead to other abilities being found to be irrelevant because of lack of attempts to engage in social activities.

In a very recent study (Strassnig, et al., 2018) we examined 554 patients with schizophrenia who had been rated for their everyday functioning and assessed with the Positive and Negative Syndrome Scale (PANSS; Kay, 1991). We used the PANSS reduced emotional experience factor identified by Khan et al. (2017) and Harvey et al (2017) to separate patients with less than moderate symptoms from patients with moderate or greater severity levels of these symptoms. We then correlated symptom severity with three elements of informant rated everyday outcomes: vocational functioning, everyday activities, and interpersonal functions. We found correlations between symptom severity and reduced interpersonal functioning in both severity subgroups, but these symptoms were minimally related to other aspects of outcome.

Because we did not have an assessment of social cognition in all of the patients in that prior study, we were not able to examine the importance of other potential predictors of impairments in social outcomes. In this report, we were able to perform those analyses with data from our multisite, multi-phase Social Cognition Psychometric Evaluation (SCOPE; Pinkham et al., 2016; 2018) study. In the SCOPE study, targeted at optimizing the assessment of social cognition, participants with schizophrenia were given a array of tests that assessed social cognition, as well as performance-based assessments of social competence. Individuals were also rated for their everyday functioning by high contact informants. The current paper reports analyses of combined data from two different phases of SCOPE, both of which included the same five social cognitive tests and employed identical strategies for the assessment of all other predictors and functional outcomes. As noted above, our hypothesis was that in patients with greater severity of reduced emotional experience, performance on social cognitive tests and measures of social competence would be less predictive of everyday functioning than in patients with lower levels of severity.

\section{Method}

\subsection{Participants}

The SCOPE Phase 3 and Phase 5 studies supply these data. Phase 3 of the study took place at Southern Methodist University (SMU; Dallas, Texas) and the University of Miami Miller 
School of Medicine (UM), while Phase 5 took place at UM, UTD, and the University of North Carolina at Chapel Hill (UNC).

The methods for diagnosis, assessment, recruitment, and exclusion were the same across the two studies. Patients underwent a clinical interview utilizing the MINI International Neuropsychiatric Inventory (Sheehan et al., 1998) and the SCID Psychosis and Substance Abuse Modules (First, et al., 2002). This evaluation was used to confirm an established clinical diagnosis of schizophrenia or schizoaffective disorder, which was a requirement for participation in the study. Another requirement was that patients had been on a consistent medication schedule for at least six weeks with no changes in the dosage of their medication in the past two weeks.

\subsection{Exclusion Criteria}

Criteria that excluded study participants included: 1) current or history of disorders, medical or neurological, that can impact brain function, 2) current or history of intellectual disability (defined as IQ $<70$ by DSM-IV criteria) or of extensive developmental disorder, 3) sensory impediments including hearing and/or visual (e.g. glaucoma, blindness, vision uncorrectable to 20/40) impairments that hinder the ability to adequately assess the participant, 4) a lack of proficiency in English, 5) a history of substance abuse in the past month, disregarding caffeine or nicotine, and 6) substance dependence that has not been in remission for at least the past six months. Patients were also excluded for hospitalizations in the past two months.

In this study, there were 397 patients with schizophrenia (52\%)/schizoaffective disorder (48\%). All participants provided signed informed consent, and this study was reviewed by the IRBs at all research sites.

\subsection{Measures}

2.3.1. Real World Functioning-A 31-item version of the Specific Levels of Functioning (SLOF; Schneider and Struening, 1983) was used to assess real world functioning. The SLOF measures social functioning, community living skills, and self-care using informant ratings. Social functioning includes social acceptability and interpersonal relationships. Community living skills included work skills and participation in activities. Ratings for participants were made by either a residential facility manager, a case manager, or another caregiver or clinician who endorsed knowing the participant "very well". Participants identified the informants. Each item on the assessment was rated using a 1 to 5 point scale. Higher scores indicate better functioning. The SLOF does not necessitate training for the interviewer or an interview with the patient.

In this study, we abbreviated the original SLOF to look at 2 socially relevant functional domains: Social Acceptability (e.g., interacting and communicating in a socially appropriate and disciplined manner) and Interpersonal Functioning (e.g., initiating, accepting and maintaining social contacts; effectively communicating). Out of the patients in Phases 3 and 5 of the study, 85 participants an informant could not identify an appropriate quality. All further analyses excluded these patients. We used the average item scores for all items because informants stated that they lacked adequate information to base their judgements on 
certain items, resulting in missing data. This strategy was consistent with the strategies in several prior studies utilizing this instrument (e.g., Harvey et al., 2011; Pinkham et al., 2016;2018).

\subsubsection{Social Skills Performance Assessment-Social Skills Performance}

Assessment (SSPA; Patterson et al., 2001). The SSPA was used to index social competence. The SSPA is a role-play evaluation tool that requires participants to start and hold a conversation in two different social situations. The two social situations included negotiating with a landlord to repair a leak and introducing yourself to a new neighbor.

The interactions were audiotaped and an expert rater who was unaware of all of the participant's other information coded the audiotaped encounter with regard to overall abilities, social appropriateness, fluency, focus, interest, and clarity. In addition, the role-play measure involving negotiating with a landlord was coded for persistence and ability. The dependent measure used in our analyses was the average score between the two role-play exercises. The score could range from 1 to 5 .

The Wide Range Achievement Test $-3^{\text {rd }}$ Edition Reading (WRAT-3; Jasatak, 1993) was used in order to estimate premorbid IQ in line with previous studies (Weickert, et al., 2000) and the scaled scores were analyzed.

\subsubsection{Reduced Emotional Experience}

Symptoms Assessment.: Severity of symptoms was evaluated with the PANSS , which was administered in its entirety by trained raters. We used these total PANSS data to develop the negative symptom model as well as to generate a rating of psychotic symptoms, which was compared across the groups defined by the severity of reduced emotional experience.

Negative Symptom Models.: A two-factor model of expression and experience was created and tested in several samples in a study by Khan et al (2017). The model as also examined for its link to functional outcomes (Harvey et al., 2017; Strassnig et al., 2018). The items in the PANSS Reduced Emotional Experience factor are: Emotional Withdrawal (N2), Passiveapathetic Social Withdrawal (N4) and Active social avoidance (G16).

As we knew that the scores for PANSS reduced expression subscale were uncorrelated with the other SLOF subscales (Harvey et al., 2017) and we knew that the PANSS Marder Factor Total scores did not add predictive variance beyond the PANSS reduced emotional experience items (Strassnig et al., 2018), we used the reduced emotional experience items alone. Then we divided the patients into two groups, based on the results described above: those with at least one of the three PANSS items scored at 4 or more (moderate reduced emotional experience symptoms) and those without a single item rated as 4 or more.

2.3.4. Social Cognitive Measures-As these measures have been described in detail previously (Pinkham et al., 2015; 2018) we describe them briefly. All tests were administered in both studies. 
Bell Lysaker Emotion Recognition Task (BLERT; Bryson et al., 1997).: The BLERT measures the ability to correctly identify seven emotional states: happiness, sadness, fear, disgust, surprise, anger, or no emotion. Participants viewed 21 10-second video clips of a male actor, providing dynamic facial, vocal-tonal, and upper-body movement cues. After viewing each video, participants identified the expressed emotion. Performance was indexed as the total number of correctly identified emotions (ranging from 0 to 21).

Penn Emotion Recognition Text (ER-40; Kohler et al., 2003).: The ER-40 includes 40 color photographs of static faces expressing 4 basic emotions (i.e. happiness, sadness, anger, or fear) and neutral expressions. Stimuli are balanced for poser's gender, age, and ethnicity, and for each emotion category, 4 high-intensity and 4 low-intensity expressions are included. Participants viewed one image at a time and chose the correct emotion label for each face. Accuracy scores, ranging from 0 to 40 , were the primary dependent variable.

Reading the Mind in the Eyes Test (Eyes; Baron-Cohen et al., 2001).: The Eyes task measures the capacity to discriminate the mental state of others from expressions in the eye region of the face. Participants viewed 36 photos of the eye region of different faces and chose the most accurate descriptor word for the thought/feeling that was portrayed. Four possible options were presented with each photo, and a glossary of mental state terms was provided for reference. The dependent measure was the total number of correct responses, ranging from 0 to 36 .

The Awareness of Social Inferences Test, Part III (TASIT; McDonald et al., 2003).: The TASIT assesses detection of lies and sarcasm. Participants watched short videos of everyday social interactions and answered four standard questions per video that probed understanding of the intentions, beliefs, and meanings of the speakers and their exchanges. Total number correct indexed performance, and scores ranged from 0 to 64 .

Hinting Task; (Corcoran et al., 1995.): The Hinting Task examines the ability of individuals to infer the true intent of indirect speech. Ten short passages present an interaction between two characters, and each passage ends with one of the characters dropping a hint. Passages were read aloud by the experimenter, and participants were asked what the character truly meant. If the first response provided was inaccurate, a second hint was delivered, allowing participants to earn partial credit for that passage. Total scores ranged from 0 to 20 .

\subsection{Data Analysis}

We compared social cognition, social competence, and reduced emotional experience, as well as demographic information across the patients in the two phases of the SCOPE study in order to determine if we needed to perform any corrections after combining the samples. After creation of the reduced emotional experience subgroups we also examined the prevalence of these subgroups in each sample, as well as the differences in everyday outcomes, social cognition, and social competence associated with the subgroups. Our final analyses were prediction of socially relevant aspects of functioning using social cognitive performance and social competence scores in the patients with lower and higher levels of 
reduced experience symptoms. For these analyses we used Pearson correlations and regression analyses. For each regression analysis, we entered the 5 social cognition variables, SSPA scores, and severity scores for reduced emotional experience in a stepwise forward entry analysis.

\section{Results}

Table 1 has the demographic information on thel patients. When the data were compared across severity of reduced emotional experience, the patients with greater severity had less education and greater severity scores for both PANSS psychosis items and for total BDI scores. There were no differences in sex, racial status, employment, independence in residence, or types of antipsychotic treatment. When we compared the patients in the two phases of the study there were statistically significant differences between the samples on total years of education and WRAT scores with SCOPE 5 patients having more education, (Scope $3 \mathrm{M}=12.73, \mathrm{SD}=2.14$; Scope $5 \mathrm{M}=13.27, \mathrm{SD}=2.38, \mathrm{t}=2.01 \mathrm{p}=.04$; and higher WRAT scores(Scope $3 \mathrm{M}=93.81, \mathrm{SD}=15.80$; Scope $5 \mathrm{M}=97.43, \mathrm{SD}=14.40, \mathrm{t}=2.08 \mathrm{p}=.038$ ). This is likely due to the addition of the UNC site which recruited less chronic patients.

Table 2 presents the scores on the social cognition measures, negative symptoms, SLOF scores, and SSPA scores in the combined samples. The SCOPE Phase 5 patients performed better on the BLERT, the ER-40, the Eyes Test, and the TASIT (all $\mathrm{t}(310)>2.32$, all $\mathrm{p}<.02$ ), but not on the hinting test, $\mathrm{t}(310)=-.42, \mathrm{p}=.67$. There were no differences in severity of reduced emotional experience or on the SSPA (all $\mathrm{t}(310<1.72$, all $\mathrm{p}>.09)$ ). SCOPE Phase 5 patients had higher scores on the SLOF subscale for interpersonal functioning, $\mathrm{t}(310)=2.79$, $\mathrm{p}=.009$, but the groups did not differ in social acceptability, $\mathrm{t}(310)=1.71, \mathrm{p}=.10$.

103 of $312(33 \%)$ cases had moderate symptoms of reduced emotional experience. $36 \%$ of the cases in SCOPE Phase 3 fell into that group, as did 30\% of the participants in Phase 5, with this difference not statistically significant, $X^{2}(1)=1.23, p=.27$. When we compared patients with a primary diagnosis of schizoaffective disorder to those with a primary diagnosis of schizophrenia on the prevalence of meeting criteria for more severe reduced emotional experience symptoms, we found that the differences were non-significant, $\mathrm{X}^{2}$ $(1)=2.05, p=.36$. When we compared patients with higher and lower levels of reduced emotional experience on the social cognitive measures and the SSPA, there were no statistically significant differences: all $\mathrm{t}(310)<1.39$, all $\mathrm{p}>$.17. SLOF scores for interpersonal functioning and social acceptability were the only two variables that differed across the reduced emotional experience groups, both $\mathrm{t}(310)>3.00, \mathrm{p}<.003$. The effect size for the difference in interpersonal functioning was quite substantial, $\mathrm{d}=.87$, while the differences for social acceptability were considerably smaller, $\mathrm{d}=.35$.

The Pearson correlations between social cognitive performance, social competence, and SLOF rating of interpersonal functioning and social acceptability are presented in Table 3. As can be seen in the table, all correlations between social cognitive measures and SLOF interpersonal functioning, other than the eyes test, were significant in patients with lower severity and none were significant in the group with higher severity (albeit with a smaller sample size; BLERT correlations were similar in the two groups). For social acceptability, 
the BLERT and the Eyes test were significant in the group with lower severity and none were significant in the group with higher severity. Again, there was one correlation, the BLERT, that would have been significant if the samples were equivalent in size.

Next, we used regression analysis to predict everyday functioning in the lower and higher reduced emotional experience severity groups. For both subgroups, we predicted the two SLOF variables in separate analyses. The results of these analyses are presented in Table 4. As we entered all of the Social cognition variables, there is a chance that there could be multicollinearity and so we present the intercorrelations of the social cognition variables in supplemental table 1 . All of the variables were intercorrelated in both groups other than the hinting task in the higher severity subsample.

All four regression analyses were statistically significant overall, all $\mathrm{F}>4.30$, all $\mathrm{p}<.04$. For interpersonal functioning in the patients with lower levels of reduced emotional experience, scores on the BLERT accounted for $9 \%$ of the variance and severity of reduced experience negative symptoms added 6\%. For interpersonal functioning in the patients with higher severity of reduced emotional experience, the only significant predictor was SSPA performance, accounting for $9 \%$ of the variance. For social acceptability in the patients with lower levels of reduced emotional experience, the hinting task was the only significant predictor, accounting for $4 \%$ of the variance. For social acceptability in the patients with higher severity of reduced emotional experience, the only significant predictor was negative symptom severity, accounting for $4 \%$ of the variance.

Since the 5 social cognition variables were all quite intercorrelated in both subgroups of patients and given that social cognitive variables in schizophrenia are often found to be unifactorial, we computed collinearity statistics for the neurocognitive predictors with the SPSS (V24) collinearity diagnostics routine. The critical statistics are "condition indices" which are computed as the square roots of the ratios of the largest eigenvalue to each successive eigenvalue. Values greater than 15 indicate a possible problem with collinearity and greater than 30 reflects a serious problem. Across the 4 regression analyses, there were no identified dimensions that exceeded the threshold of 15 and the largest condition index was 7.49 .

\section{Discussion}

In our large sample of people with schizophrenia, 33\% had evidence of moderate levels of reduced emotional experience, which is consistent with previous estimates of the prevalence of persistent negative symptoms across several different rating methods in clinically stable patients (Rabinowitz et al., 2013). The patients with higher levels of reduced emotional experience had evidence of more social impairments and within that group, performance on tests of social cognitive ability did not predict social outcomes. In patients with lower levels of reduced emotional experience symptoms, social cognitive abilities, in specific emotion recognition ability and mentalizing test performance, predicted interpersonal functioning and disruptive interpersonal behavior respectively. It is of interest that the prevalence and severity of reduced emotional expression symptoms did not differ across two samples from different phases of the study despite the fact that the samples varied in their academic and 
intellectual performance. Further, social cognitive abilities did not differ at all as a function of negative symptoms of reduced emotional experience, a finding confirmed by the observation that social cognitive abilities and symptom severity combined to predict independent variance in interpersonal functioning limitations in patients with lower levels of reduced emotional experience.

These data suggest that previously validated treatments (Horan et al., 2009; Combs et al., 2007; Kurtz et al., 2016) aimed at social cognitive deficits may not have the same efficacy for improving real world social outcomes in patients with more severe symptoms of reduced emotional experience. Likewise, social competence predicted social outcomes more efficiently in the patients with greater negative symptom severity and social cognition did not contribute. Thus, social skills interventions may be important for this subgroup of patients in particular (Kurtz and Mueser, 2008). It has been suggested that social skills training may directly reduce the emotional deficits seen in schizophrenia (Turner et al., 2018) and the higher severity subgroup may be a particularly relevant group to treat (Granholm and Harvey, 2018).

Previous studies have suggested that negative symptoms may have more importance for the prediction of nonsocial everyday outcomes than we have found our recent studies. In specific, negative symptoms early in the course of illness may exert a longitudinal adverse impact on other aspects of functioning (Ventura et al., 2015) through reductions in motivation and other associated interpersonal limitations.

There are some limitations in this study. Patients were not selected on the basis of their negative symptom severity and people with truly severe negative symptoms are underrepresented. Further, patients with very severe negative symptoms are likely to be underrepresented in all studies where office visits and spontaneous decisions to participate in research are made. The social cognitive tests we examined were found to predict functional outcomes, but the individual contributions of these tasks to prediction of functional outcomes is small. Finally, active social avoidance is controversial as a negative symptom. At levels of severity greater than that seen in any patient in this sample (i.e., PANSS item score $=7$ ), the PANSS definition of active social avoidance explicitly refers to being influenced by delusions. Lower levels of severity refer to anxiety and fear as motivators for active avoidance.

In summary, reduced emotional experience, social cognition, and social competence exert generally independent and cumulative influences on social outcomes. These influences are different depending on the level of severity of reduced emotional experience and these symptoms appear to be important for the prediction of social outcomes at both low and high levels of severity.

\section{Supplementary Material}

Refer to Web version on PubMed Central for supplementary material. 


\section{Acknowledgments}

Role of Funding Source

The data in this study were funded by NIMH Grant 93432 to Drs. Harvey, Penn, and Pinkham. The NIMH had no role in the preparation of this paper.

Dr. Jarskog has received research grant funding from NIH, Auspex/Teva, Boehringer-Ingelheim and Otsuka.

\section{References}

Baron-Cohen S, Wheelwright S, Hill J, Raste Y, Plumb I,2001 The 'Reading the mind in the eyes' Test revised version: A study with normal adults, and adults with Asperger syndrome or high-functioning autism. J. Child Psychol. Psychiatr. 42(2),241-251.

Bryson G, Bell M, Lysaker P,1997 Affect recognition in schizophrenia: a function of global impairment or a specific cognitive deficit. Psychiatr. Res. 71(2),105-113.

Carrión RE, Demmin D, Auther AM, et al. 2016 Duration of attenuated positive and negative symptoms in individuals at clinical high risk: Associations with risk of conversion to psychosis and functional outcome. J. Psychiatric Res. 81,95-101.

Combs DR, Adams SD, Penn DL, Roberts D, Tiegreen J, Stern P,2007 Social Cognition and Interaction Training (SCIT) for inpatients with schizophrenia spectrum disorders: preliminary findings. Schizophr. Res. 91,112-116. [PubMed: 17293083]

Corcoran R, Mercer G, Frith CD,1995 Schizophrenia, symptomatology and social inference: investigating "theory of mind" in people with schizophrenia. Schizophr. Res. 17(1),5-13. [PubMed: 8541250]

First B, Spitzer RL, Gibbon M, and Williams JBW,2002 Structured Clinical Interview for DSM-IV-TR Axis I Disorders, Research Version, Non-patient Edition (SCID-I/NP) New York: Biometrics Research, New York St Psychiatric Inst.

Galderisi SA, Rossi A, Rocca P, Bertolino A, et al.,2014 The influence of illness-related variables, personal resources and context-related factors on real-life functioning of people with schizophrenia. World Psychiatr. 13,275-287.

Granholm E, Harvey PD,2018 Social Skills Training for Negative Symptoms of Schizophrenia. Schizophr. Bull (44),472-474. [PubMed: 29315427]

Harvey PD, Khan A, Keefe RSE,2017 Using the Positive and Negative Syndrome Scale (PANSS) to Define Different Domains of Negative Symptoms: Prediction of Everyday Functioning by Impairments in Emotional Expression and Emotional Experience. Innov. Clin. Neurosci. 14,18-22. [PubMed: 29410933]

Harvey PD, Raykov T, Twamley EW, Vella L, Heaton RK, Patterson TL, 2011 Validating the measurement of real-world functional outcome: phase I results of the VALERO study. Am. J. Psychiatry 168, 1195-2001. [PubMed: 21572166]

Horan WP, Kern RS, Shokat-Fadai K, et al.,2009 Social cognitive skills training in schizophrenia: an initial efficacy study of stabilized outpatients. Schizophr. Res. 107,47-54. [PubMed: 18930378]

Jastak S: Wide-Range Achievement Test, 3rd ed. San Antonio, TX, Wide Range, Inc, 1993

Kalin M, Kaplan S, Gould F, et al.,2015 Social cognition, social competence, negative symptoms and social outcomes: Inter-relationships in people with schizophrenia. J. Psychiatric Res. 68,254-60.

Khan A, Liharska L, Harvey PD, Atkins A, Ulshen D, Keefe RSE 2017 Negative Symptom Dimensions of the Positive and Negative Syndrome Scale Across Geographical Regions: Implications for Social, Linguistic, and Cultural Consistency. Innov. Clin. Neurosci. 14:30-40.

Kohler CG, Turner TH, Bilker WB, et al.,2003 Facial emotion recognition in schizophrenia: intensity effects and error pattern. Am. J. Psychiatr. 160,1768-1774. [PubMed: 14514489]

Kurtz MM, Gagen E, Rocha NBF, Machado S, \& Penn DL 2016 Comprehensive, standalone treatments for social cognitive deficits in schizophrenia: A critical review and effect-size analysis of controlled studies. Clin. Psychol. Rev, 43, 80-89. [PubMed: 26437567] 
Kurtz MM, Mueser KT,2008 A meta-analysis of controlled research on social skills training for schizophrenia. J. Consult Clin. Psychol. 76,491-504. [PubMed: 18540742]

Lindenmayer JP, Khan A, McGurk SR, et al., 2018 Does social cognition training augment response to computer-assisted cognitive remediation for schizophrenia? Schizophr. Res. In press.

Lindenmayer JP, McGurk SR, Khan A, et al.,2013 Improving social cognition in schizophrenia: a pilot intervention combining computerized social cognition training with cognitive remediation. Schizophr. Bull 39(3),507-517. [PubMed: 23125396]

McDonald S, Flanagan S, Rollins J, Kinch J, 2003 TASIT: A new clinical tool for assessing social perception after traumatic brain injury. J. Head Trauma Rehab. 18(3), 219-238.

Nuechterlein KH, Green MF, Kern RS, et al.,2008 The MATRICS Consensus Cognitive Battery, part 1: test selection, reliability, and validity. Am. J. Psychiatr. 165(2),203-213. [PubMed: 18172019]

Pinkham AE, Harvey PD, Penn DL,2018 Social Cognition Psychometric Evaluation: Results of the Final Validation Study. Schizophr. Bull 44, 737-748 [PubMed: 28981848]

Pinkham AE, Penn DL, Green MF, Harvey PD,2016 Social Cognition Psychometric Evaluation: Results of the Initial Psychometric Study. Schizophr. Bull 42(2),494-504. [PubMed: 25943125]

Rabinowitz J, Werbeloff N, Caers I, Mandel FS, Stauffer V, Mernard F, Kinon BJ, Kapur S,2013 Negative symptoms in schizophrenia - the remarkable impact of inclusion definitions in clinical trials and their consequences. Schizophr. Res. 150(2-3),334-338. [PubMed: 23815975]

Raine A,2006 Schizotypal personality: neurodevelopmental and psychosocial trajectories. Annu Rev Clin Psychol. 2,291-326. [PubMed: 17716072]

Schneider LC, Struening EL, 1983 SLOF: a behavioral rating scale for assessing the mentally ill. Soc. Work Res. Abstr. 19(3), 9-21. [PubMed: 10264257]

Sergi MJ, Fiske AP, Horan WP, et al., 2009 Development of a measure of relationship perception in schizophrenia. Psychiatr. Res. 166,54-62.

Sheehan DV, Lecrubier Y, Harnett-Sheehan K, et al.,1998 The mini international neuropsychiatric interview (M.I.N.I.): The development and validation of a structured diagnostic psychiatric interview. J. Clin. Psychiatr. 59, 22-33.

Strassnig MT, Bowie CR, Pinkham AE, et al., 2018 Which levels of cognitive impairments and negative symptoms are related to functional deficits in schizophrenia? J. Psychiatric. Res, 104, 124-129.

Strassnig MT, Raykov T, O’Gorman C, et al.,2015 Determinants of Different Aspects of Everyday Outcome in Schizophrenia: The Roles of Negative Symptoms, Cognition, and Functional Capacity. Schizophr. Res. 165,76-82. [PubMed: 25868935]

Strauss GP, Horan WP, Kirkpatrick B, et al.,2013 Deconstructing negative symptoms of schizophrenia: avolition-apathy and diminished expression clusters predict clinical presentation and functional outcome. J. Psychiatric Res. 47,783-9.

Turner DT, McGlanaphy E, Cuijpers P, van der Gaag M, Karyotaki E,2018 A Meta-Analysis of Social Skills Training and Related Interventions for Psychosis. Schizophr. Bull 44: 475-491 [PubMed: 29140460]

Ventura J, Subotnik KL, Gitlin MJ, et al., 2015 Negative symptoms and functioning during the first year after a recent onset of schizophrenia and 8 years later. Schizophr. Res. 161, 407-411 [PubMed: 25499044]

Weickert TW, Goldberg TE, Gold JM, Bigelow LB, Egan MF, Weinberger DR.(2000). Cognitive impairments in patients with schizophrenia displaying preserved and compromised intellect. Arch. Gen. Psychiatry. ;57(9):907-913. [PubMed: 10986554] 


\section{Table 1}

Demographic and Treatment Information By Severity of Reduced Emotional Experience

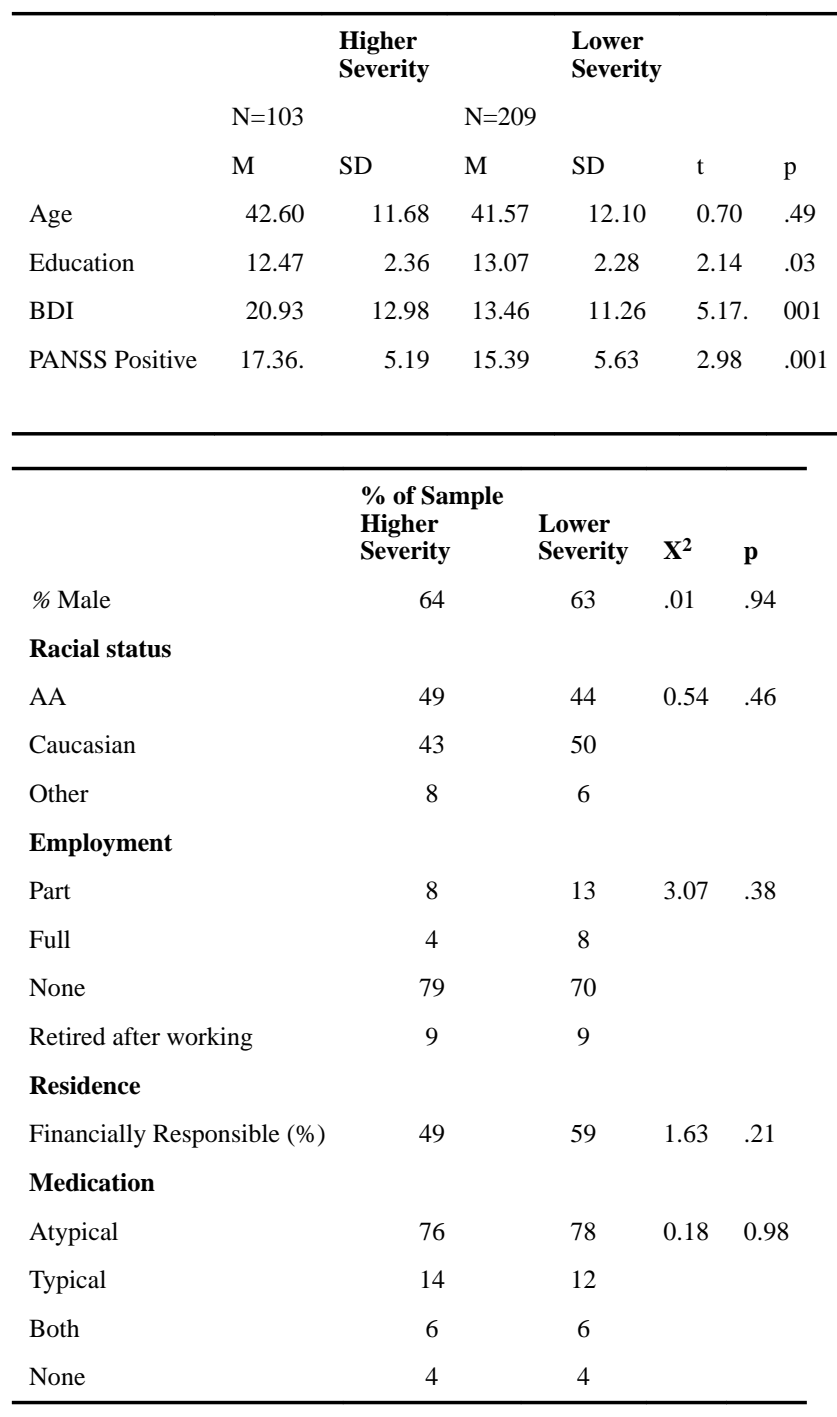


Table 2

Scores on Social Cognition, Social Competence, Negative Symptoms, and Everyday Functioning

\begin{tabular}{|c|c|c|c|}
\hline & \multicolumn{2}{|c|}{$(\mathrm{N}=312)$} & \multirow[b]{2}{*}{ Possible Range } \\
\hline & $\mathbf{M}$ & SD & \\
\hline BLERT & 14.00 & 3.83 & $0-21^{a}$ \\
\hline ER-40 & 30.66 & 5.03 & $0-40^{a}$ \\
\hline Eyes & 21.24 & 5.51 & $0-36^{a}$ \\
\hline Hinting & 13.77 & 3.58 & $0-20^{a}$ \\
\hline TASIT & 45.31 & 7.35 & $0-64^{a}$ \\
\hline Emotional Withdrawal & 1.69 & 1.08 & $1-7^{b, c}$ \\
\hline Passive Social Withdrawal & 2.37 & 1.45 & $1-7^{b, c}$ \\
\hline Active Social Avoidance & 2.33 & 1.46 & $1-7^{b, c}$ \\
\hline SSPA Scores & 4.23 & .57 & $0-5^{a}$ \\
\hline SLOF Interpersonal & 3.39 & .90 & $1-5^{a, c}$ \\
\hline SLOF Social Acceptability & 4.37 & .57 & $1-5^{a, c}$ \\
\hline \multicolumn{4}{|l|}{ Higher is better } \\
\hline Higher is worse & & & \\
\hline
\end{tabular}


Table 3

Pearson Correlations between Social Cognition, Social Competence, and Everyday Social outcomes

\begin{tabular}{|c|c|c|}
\hline \multicolumn{3}{|c|}{$\begin{array}{l}\text { Lower Reduced emotional experience Symptoms } \\
\text { (N=209) }\end{array}$} \\
\hline & SLOF Interpersonal & SLOF Social Acceptability \\
\hline BLERT & $.29^{* *}$ & $.14^{*}$ \\
\hline ER-40 & $.14^{*}$ & .04 \\
\hline Eyes & .10 & $.15^{*}$ \\
\hline Hinting & $.21^{*}$ & .01 \\
\hline TASIT & $.17^{*}$ & .06 \\
\hline SSPA & $.18^{*}$ & -.04 \\
\hline \multicolumn{3}{|c|}{$\begin{array}{l}\text { Higher Reduced emotional experience Symptoms } \\
(\mathbf{n = 1 0 3})\end{array}$} \\
\hline & SLOF Interpersonal & SLOF Social Acceptability \\
\hline BLERT & .14 & .17 \\
\hline ER-40 & .04 & -.05 \\
\hline Eyes & -.06 & .05 \\
\hline Hinting & .01 & .05 \\
\hline TASIT & .10 & -.07 \\
\hline SSPA & $.31 *$ & .04 \\
\hline
\end{tabular}




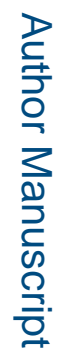

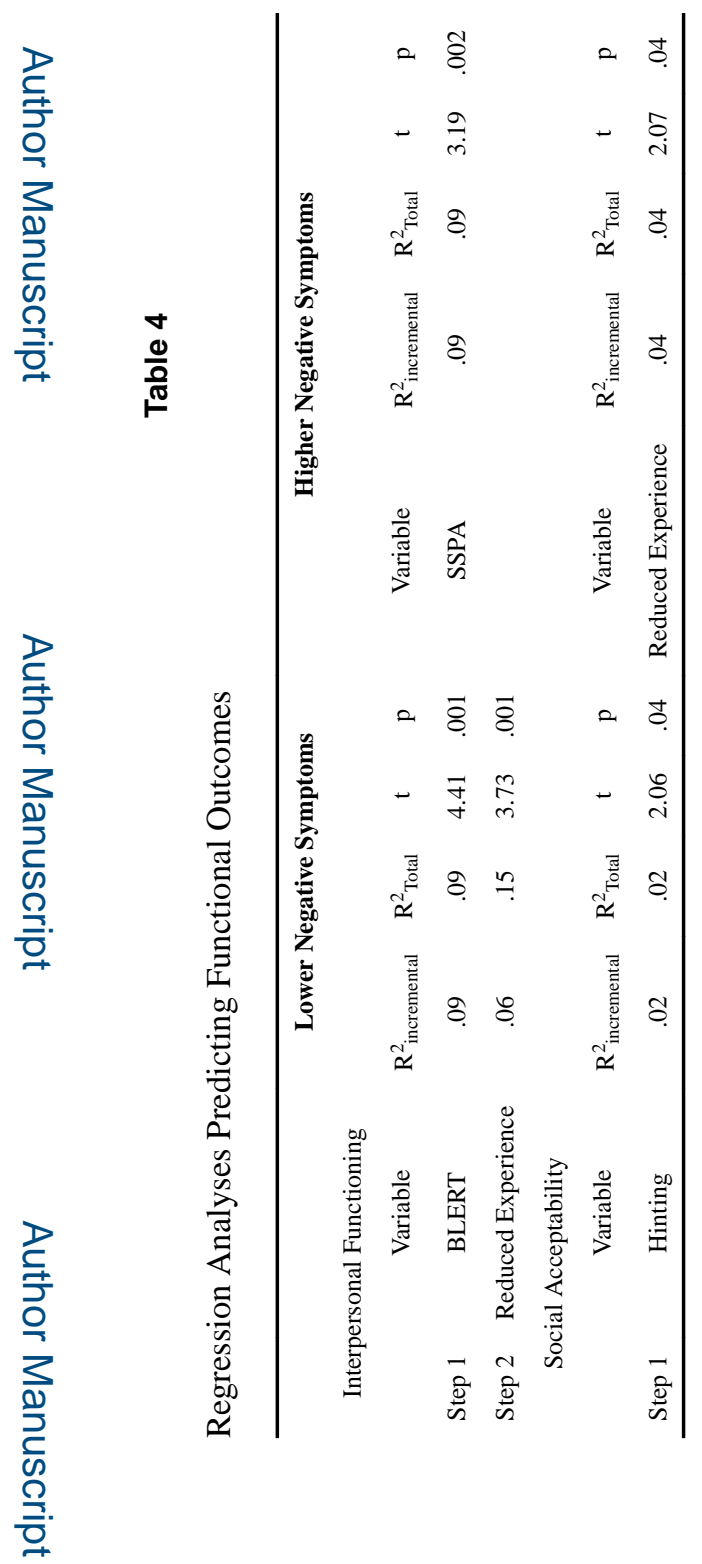

Schizophr Res. Author manuscript; available in PMC 2020 April 01. 